\title{
Uso da avaliação multidimensional em idosos com Diabetes mellitus na Atenção Primária à Saúde: um estudo piloto
}

\author{
Juliete Coelho Gelsleuchter ${ }^{\mathrm{a}^{*}}$, Karina Silveira de Almeida Hammerschmidt ${ }^{\mathrm{b}}$, Juliana \\ Balbinot Reis Girondi ${ }^{\mathrm{a}}$, Melissa Orlandi Honório Locks ${ }^{\mathrm{a}}$, Laura Cavalcanti de Farias \\ Brehmer $^{\mathrm{a}}$, Anderson Abreu de Carvalho
}
aPrograma de Pós-Graduação em Enfermagem, Universidade Federal de Santa Catarina (UFSC), Florianópolis, SC, Brasil

bUniversidade Federal do Paraná (UFPR), Curitiba, PR, Brasil.

'Programa de Pós-Graduação em Gestão do Cuidado em Enfermagem, Universidade Federal de Santa Catarina

(UFSC), Florianópolis, SC, Brasil.

Histórico do Artigo: Recebido em: 24/08/2020

Aceito em:

$03 / 11 / 2020$

Palavras-chave:

Cuidado de enfermagem; saúde do idoso; diabetes mellitus: gerontologia; atenção primária à saúde

Keywords: Nursing care; elderly health; diabetes mellitus; gerontology; primary health care.

\begin{abstract}
RESUMO
O diabetes mellitus representa importante causa de morbidade e mortalidade na população idosa, associado a hábito alimentares inadequados, sedentarismo e fatores socioeconômicos. Aspectos preventivos e o tratamento adequado são fundamentais para a manutenção da qualidade de vida nesses indivíduos. Assim, objetiva-se identificar os itens da avaliação multidimensional utilizados na consulta de enfermagem ao idoso com diabetes mellitus. Trata-se de estudo piloto do tipo caso múltiplo holístico, conduzido em 19 idosos diagnosticados com diabetes mellitus e usuários da Unidade Básica de Saúde de São José, Santa Catarina. Brasil. A coleta de dados ocorreu em 2017 e deu-se por análise descritiva analítica documental em prontuários. A idade dos idosos variou entre 61 e 82 anos, sendo 7 mulheres e 12 homens. Hipertensão e dislipidemia foram as comorbidades mais prevalentes. Os itens da avaliação multidimensional mais prevalentes foram: verificação da pressão arterial e peso $(73,6 \%)$ e verificação de glicemia capilar $(36,8 \%)$. Os seguintes itens da avaliação estiveram ausentes nos prontuários: alimentação e nutrição; acuidade visual; acuidade auditiva; incontinência urinaria; sexualidade; vacina; avaliação cognitiva; depressão; mobilidade; queda; avaliação funcional; e, suporte familiar. Também, foi possível identificar itens relacionados ao registro de enfermagem, com destaque para a falta de identificação e escassez de registros, e itens da avaliação multidimensional do idoso, usados nas práticas dos enfermeiros através dos registros. Neste contexto, foi evidenciado o baixo uso da avaliação multidimensional para o idoso com diabetes mellitus e a escassez dos registros de enfermagem.
\end{abstract}

The use of multidimensional assessment in elderly people with Diabetes mellitus in Primary Health Care: a pilot study

\section{ABSTRACT}

Diabetes mellitus represents an important cause of morbidity and mortality in the elderly population, associated with inappropriate eating habits, physical inactivity and socioeconomic factors. Preventive aspects and adequate treatment are essential for maintaining the quality of life in these individuals. Thus, the objective is to identify the items of the multidimensional evaluation used in the nursing consultation for the elderly with diabetes mellitus. This is a holistic, multi-case pilot study approach, conducted in 19 elderly people diagnosed with diabetes mellitus and users of the Basic Health Unit of São José, Santa Catarina. Brazil. Data collection took place in 2017 and was carried out through documentary analytical descriptive analysis in medical records. The age of the elderly ranged between 61 and 82 years, with 7 women and 12 men. Hypertension and dyslipidemia were the most prevalent comorbidities. The items most found in the multidimensional evaluation were: checking blood pressure and weight $(73.6 \%)$ and checking blood glucose $(36.8 \%)$. The following items of the evaluation were absent from the medical records: food and nutrition; visual acuity; auditory acuity; urinary incontinence; sexuality; vaccine; cognitive assessment; depression; mobility; fall; functional assessment; and, family support. It was also possible to identify items related to the nursing record, with emphasis on the lack of identification and scarcity of records, and items from the multidimensional assessment of the elderly, used in nurses' practices through the records. In this context, the low use of multidimensional assessment in the elderly with Diabetes and the scarcity of nursing records was evidenced.

\footnotetext{
*Autor correspondente: julietecoelholll@gmail.com (Gelsleuchter J. C.)
} 


\section{Introdução}

Graças aos avanços científicos e tecnológicos, verifica-se aumento gradual da expectativa de vida. Em contrapartida, o envelhecimento também traz consigo maior prevalência de doenças crônico-degenerativas, com destaque para as morbidades cardiovasculares, que representam a principal causa de morte no Brasil (1).

Destacam-se entre as doenças crônico-degenerativas o diabetes mellitus, sendo importante causa de morbidade e mortalidade, especialmente entre os idosos. Com o envelhecimento populacional, observa-se maior tendência ao sedentarismo, a hábitos alimentares inadequados, fatores sócio-comportamentais, e o consequente aumento da incidência e prevalência do diabetes, incluindo a mortalidade pela doença. Estudos apontam que no ano 2000 havia 171 milhões de pessoas com diabetes no mundo, podendo em 2030 atingir 366 milhões de indivíduos. Neste cenário, o Brasil terá cerca de 11,3 milhões de pessoas com diabetes $(2,3)$.

O envelhecimento populacional é tema mundial de estudo. No Brasil, após a aprovação do pacto de gestão em fevereiro de 2006, a saúde do idoso entrou no rol das prioridades do Ministério da Saúde, buscando a atenção integral principalmente na Atenção Primária à Saúde (4). Neste contexto, destaca-se a ação de equipe interdisciplinar, visando o atendimento integral de toda população. Os idosos se caracterizam como o público que mais procura esse serviço, embora o atendimento direcionado a estes não seja específico. A população idosa necessita de atenção integral, visando a prevenção, tratamento, reabilitação e busca ativa de riscos e danos, com enfoque na melhora das condições de vida (5).

O Ministério da Saúde elaborou em seu Caderno 19 a "Avaliação Multidimensional da Pessoa Idosa", consistindo na forma de mensuração dos aspectos gerais de saúde e sociais. Esta avaliação pode ser usada por qualquer profissional da saúde, desde que capacitado, sendo preconizado o seu uso nas Unidades Básicas de Saúde. Trata-se de avaliação rápida que pode ser utilizada na identificação de problemas de saúde condicionantes de declínio funcional em pessoas idosas. Neste cenário, a enfermagem atua identificando particularidades que ocorrem em consequência do processo de envelhecimento, caracterizando riscos e prevenindo patologias. O processo de cuidado é dinâmico e ativo na resolução de problemas de saúde que acometem os idosos, além de envolver a família no cuidado.

Ressalta-se que o cuidado de enfermagem, através da consulta de enfermagem, é a referência do processo de trabalho, focando-se na intenção de atuação junto ao idoso, propondo subsídios para o envelhecimento saudável. Condizente com este contexto, emerge o objetivo de identificar os itens da avaliação multidimensional utilizados na consulta de enfermagem ao idoso com Diabetes.

\section{Materiais e Métodos}

Trata-se de um estudo piloto do tipo caso múltiplo holístico, baseado nos pressupostos de Yin (6). As variáveis do estudo são: sexo, idade, comorbidades, além dos registros de enfermagem no prontuário, como peso, altura, pressão, glicemia, entre outros, especificados a seguir no instrumento de coleta de dados.

A coleta de dados ocorreu em 2017 em uma Unidade Básica de Saúde (UBS) da região metropolitana de São José, Santa Catarina. Para tanto, foram convidados idosos com diagnóstico de diabetes que frequentaram a UBS por demanda espontânea ou por consulta agendada, durante um mês, para participarem da pesquisa após a explicação dos objetivos do estudo e o preenchimento do Termo de Consentimento Livre e Esclarecido (TCLE). 
Posteriormente, a pesquisadora teve acesso aos prontuários dos pacientes, que foram analisados criteriosamente, buscando-se as evoluções da enfermagem em relação aos registros do uso da avaliação multidimensional do idoso. Como critérios de inclusão, foram considerados: idosos acima de 60 anos; e, prontuários de idosos acima de 60 anos com diagnóstico de diabetes mellitus atendidos por enfermeiros na Unidade Básica. Ainda, os critérios de exclusão adotados foram: idosos que não tinham o diagnóstico de diabetes mellitus; e, pacientes que não possuíam evolução de enfermagem.

A coleta de dados foi realizada na própria UBS referência dos idosos, mediante utilização de instrumento elaborado pelos pesquisadores, contendo dados gerais como: data; horário; número do prontuário; iniciais do paciente; idade; comorbidades; profissional responsável pelo atendimento e dados específicos da avaliação multidimensional embasados no Caderno 19 de Atenção Básica do Ministério da Saúde (peso; pressão arterial; glicemia; nutrição; visão; audição; incontinência; atividade sexual; humor/depressão/cognição memória/exame mental; função dos membros superiores; função dos membros inferiores; atividades da vida diária; domicílio; quedas, suporte social, mobilidade e medicamentos). Cada item identificado foi registrado pela pesquisadora. Os dados coletados foram inseridos em planilha específica em software Word. As informações foram analisadas segundo análise descritiva analítica documental em prontuários.

Os aspectos éticos desta pesquisa estão de acordo com a resolução 466/12, que orienta o desenvolvimento de pesquisas com seres humanos no Brasil. O protocolo de pesquisa foi aprovado pelo Comitê de Ética em Pesquisa da Universidade Federal de Santa Catarina (parecer 1.833.375) em conjunto com aprovação da Secretária de Saúde de São José, Santa Catarina, Núcleo de Educação Permanente. Para preservar o anonimato dos participantes, os mesmos foram identificados e tiveram seus nomes associados com plantas medicinais.

\section{Resultados}

Foram avaliados prontuários de 19 idosos com diabetes mellitus, todos usuários da UBS de São José, Santa Catarina. Prevaleceram prontuários de idosos com idade entre 61 e 82 anos, média de idade de 70 anos, sendo $12(63,1 \%)$ do gênero masculino e 7 $(36,9 \%)$ feminino. Destacaram-se como comorbidades prevalentes ao diabetes mellitus, a hipertensão sistêmica e a dislipidemia. Com intuito de melhor caracterizar o perfil destes participantes, dados coletados são apresentados a seguir (Tabela 1).

Verificou-se nos registros da enfermagem a presença de itens relacionados a avaliação multidimensional: verificação de peso e pressão arterial em $73,6 \%$ dos prontuários, havendo 13 verificações de pressão arterial e um controle de pressão arterial (realizado três vazes na semana, por um mês, na Unidade de Saúde). Quanto à verificação de glicemia capilar, foi identificado em $36,8 \%$ dos prontuários. Desses, seis foram verificações pontuais e um controle glicêmico. A verificação da temperatura corporal foi identificada em $10,5 \%$ dos prontuários e a estatura e relato de dificuldade de cicatrização em 5,2\% dos casos (Tabela 2). 
Tabela 1 - Caracterização do perfil e comorbidades prevalentes em idosos usuários da Unidade Básica de Saúde de São José, Santa Catarina, 2017.

\begin{tabular}{|c|c|c|c|}
\hline Iniciais Identificação & Sexo & Idade & Comorbidades \\
\hline Alecrim & Feminino & 82 & $\begin{array}{c}\text { Hipertensão } \\
\text { Dislipidemia }\end{array}$ \\
\hline Arruda & Feminino & 71 & $\begin{array}{l}\text { Hipertensão } \\
\text { Dislipidemia }\end{array}$ \\
\hline Boldo & Masculino & 63 & $\begin{array}{l}\text { Transtorno Bipolar } \\
\text { Dislipidemia }\end{array}$ \\
\hline Camomila & Feminino & 67 & $\begin{array}{l}\text { Hipertensão } \\
\text { Hipertensão }\end{array}$ \\
\hline Canela & Masculino & 70 & $\begin{array}{l}\text { Esteatose } \\
\text { Dislipidemia }\end{array}$ \\
\hline Carqueja & Feminino & 66 & $\begin{array}{l}\text { Hipertensão } \\
\text { Dislipidemia }\end{array}$ \\
\hline Cavalinha & Masculino & 80 & $\begin{array}{c}\text { Hipertensão } \\
\text { Insuficiência Renal }\end{array}$ \\
\hline Citronela & Masculino & 75 & $\begin{array}{l}\text { Hipertensão } \\
\text { Dislipidemia }\end{array}$ \\
\hline Cominho & Feminino & 65 & - \\
\hline Erva-cidreira & Masculino & 72 & $\begin{array}{l}\text { Hipertensão } \\
\text { Parkinson }\end{array}$ \\
\hline Erva-doce & Masculino & 78 & $\begin{array}{l}\text { Hipertensão } \\
\text { Dislipidemia }\end{array}$ \\
\hline Gengibre & Feminino & 61 & Obesidade \\
\hline Hibiscus & Masculino & 72 & - \\
\hline Hortelã & Masculino & 66 & - \\
\hline Macela & Masculino & 77 & Hipertensão \\
\hline Malva & Masculino & 64 & $\begin{array}{c}- \\
\text { Hipotan }{ }^{2}\end{array}$ \\
\hline Pitanga & Feminino & 69 & $\begin{array}{c}\text { Hipertensão } \\
\text { Gastrite } \\
\text { Hipotireoidismo } \\
\text { Câncer de Pele } \\
\text { Bronquite }\end{array}$ \\
\hline Quebra pedra & Masculino & 61 & Hipertensão \\
\hline Unha-de-gato & Masculino & 64 & $\begin{array}{c}\text { Hipertensão } \\
\text { Arritmia Cardíaca }\end{array}$ \\
\hline
\end{tabular}

Os itens da avaliação multidimensional não encontrados nos prontuários foram: visão, audição, incontinência esfincteriana, atividade sexual, humor/comportamento/depressão, cognição, memória, exame mental, função dos MMSS, função dos MMII, atividades diárias, domicílio e quedas.

Além disso, foram identificadas algumas dificuldades nos registros dos prontuários, tais como: escassez de registros da enfermagem, registros sem identificação, prontuários sem registro de nenhum profissional, registros apenas com assinatura (rubrica), prontuários sem número de identificação e prontuários não encontrados. 
Tabela 2 - Itens da avaliação multidimensional identificados nos prontuários dos idosos usuários da Unidade Básica de Saúde de São José, Santa Catarina, 2017.

\begin{tabular}{cccc}
\hline & \multicolumn{3}{c}{ Avaliação multidimensional de idosos } \\
& Itens encontrados & $\begin{array}{c}\text { Número } \\
\text { de vezes }\end{array}$ & Forma encontrada \\
\hline Nutrição & Peso & 14 & Verificações \\
& Estatura & 1 & Verificação \\
& Pressão arterial & 14 & 1 controle; 13 verificações \\
Sistemas cardiovascular, & Dificuldade de cicatrização & 1 & $\begin{array}{c}\text { Verificação ao analisar } \\
\text { ferimento }\end{array}$ \\
& Tegumentar e circulatório & Verificações \\
& HGT & 2 & 1 controle; 6 verificações \\
\hline
\end{tabular}

\section{Discussão}

Conforme observado no presente estudo, a partir dos prontuários analisados, o perfil clínico da maioria dos usuários da UBS é composto por idosos, do sexo masculino. Interessante, a maioria dos estudos no Brasil e no mundo apontam o sexo feminino como predominante, considerando a associação de inúmeros fatores, como a maior cautela e cuidado à saúde no decorrer da vida. Ainda, estudos epidemiológicos desenvolvidos em outros estados (Pará, São Paulo e Amapá) evidenciam uma maior prevalência em mulheres $(7,8)$. Em contrapartida, pesquisa realizada no estado de Alagoas (9) apontou a prevalência de 53,59\% do sexo masculino, corroborando com os achados deste trabalho.

Em relação a idade dos idosos, pesquisa realizada no sul do Brasil, indicou a idade variando de 60 a 70 anos como a mais prevalente (10). Dentre as morbidades mais prevalentes ao diabetes mellitus, estão a hipertensão e a dislipidemias, convergindo com os achados de outros estudos $(10,11)$

O diabetes mellitus é classificado como epidemia mundial, estimando-se incidência da doença em 387 milhões de indivíduos, podendo acometer 471 milhões até 2035. O envelhecimento populacional está ligado à maior incidência de diabetes, associado a outros fatores como: maior urbanização, progressiva prevalência de obesidade e sedentarismo, bem como da maior sobrevida de pacientes com a doença (3).

O diabetes mellitus é doença crônica não transmissível, normalmente silenciosa, sendo às vezes diagnosticada tardiamente, com surgimento de alguma complicação decorrente, em especial a hipoglicemia, hiperglicemia, cetoacidose diabética, complicações microvasculares (pequenos vasos sanguíneos) e macrovasculares (grandes artérias) de elevada morbimortalidade, que podem ocorrer em curto, médio e longo prazo. Ainda, dentre as complicações mais frequentes associadas ao diabetes estão as retinopatias, nefropatias, neuropatias e doenças cardiovasculares como a doença arterial coronariana, o acidente vascular cerebral isquêmico (AVC) e a doença arterial periférica. Os portadores de diabetes mellitus requerem cuidados contínuos em saúde a fim de prevenir complicações agudas e redução do risco de complicações crônicas (12).

Conforme dados de estudo transversal realizado com 1320 usuários brasileiros com diabetes mellitus no Centro Oeste mineiro, indivíduos que possuem o diagnóstico da doença há mais de 10 anos apresentaram 156 complicações em relação àqueles que possuem o diagnóstico há menos de 10 anos, com 45 complicações, mostrando a associação direta entre as complicações e o tempo de duração da doença (13).

Importante ressaltar, pacientes com diabetes tipo II possuem maior risco de desenvolver dislipidemia, pois a resistência à insulina predispõe a alterações no metabolismo das lipoproteínas circulantes. Além disso, fatores genéticos, 
socioeconômicos e o estilo de vida sedentário também são relevantes no surgimento de dislipidemia $(14,15)$.

Nesse contexto, o alto índice de pacientes com diabetes tipo II e hipertensão está fisiologicamente associado à resistência à insulina que promove estresse oxidativo, atuando sobre o sistema nervoso simpático, responsável por estimular a musculatura lisa e retenção de sódio, elevando assim a pressão arterial. Ainda, este mecanismo patológico destaca o papel excitatório da hiperglicemia sobre o sistema reninaangiotensina-aldosterona $(16,17)$.

Todavia, os idosos são os que mais sofrem com a diabetes mellitus e suas complicações. Por apresentarem características específicas à idade, a população senescente requer maior atenção nos cuidados em saúde, fazendo-se necessária a avaliação cuidadosa que eventualmente possa identificar problemas além das queixas atuais. Portanto, o uso da avaliação multidimensional do idoso nos atendimentos em saúde, é ferramenta capaz de enriquecer o cuidado e auxiliar na detecção e resolução de problemas relacionados ao diabetes.

A avaliação multidimensional possibilita o levantamento de dados de todas as dimensões do idoso, possibilitando condutas de prevenção, orientação e encaminhamentos, objetivando melhorar a qualidade de vida desta população e procurando manter suas funções por um maior período de tempo $(18,19)$.

O Ministério da Saúde, no Caderno de Atenção Básica número 19, discorre sobre a Avaliação Multidimensional do idoso nos seguintes quesitos: alimentação e nutrição; acuidade visual; acuidade auditiva; incontinência urinaria; sexualidade; vacina; avaliação cognitiva; depressão; mobilidade; queda; avaliação funcional; e, suporte familiar. A avaliação é feita por meio de testes rápidos e por escalas (4). No presente estudo, os principais itens de avaliação multidimensional do idoso identificados nos prontuários foram: verificação de peso e verificação de pressão arterial, seguidos pela quantificação da glicemia capilar.

A avaliação antropométrica compreende método simples, porém útil para o diagnóstico nutricional dos idosos. Com o avanço da idade, ocorre o declínio da estatura em decorrência da compreensão vertebral, alterações posturais e perda do tônus muscular. Assim, torna-se importante também o controle da pressão arterial e da glicemia capilar de forma sistematizada, evitando o acometimento por doenças e preservando a capacidade funcional do indivíduo (4).

Desta forma, o uso da avaliação multidimensional no cuidado com o idoso torna-se fundamental, pois fornece subsídios para avaliação e implementação de medidas que possam evitar a perda de autonomia do paciente, ou que ao menos possam adiar essa perda por um maior tempo. Além disso, a avaliação fornece substratos na detecção de possíveis problemas, riscos, agravos e prevenção de desfechos em relação a aspectos do envelhecimento, fundamentais para prescrever o cuidado do paciente idoso com diabetes.

A Atenção Primária à Saúde por ser porta de acesso aos serviços de saúde, deste modo o uso da avaliação multidimensional do idoso possibilita identificar potencialidades e fragilidades do processo de envelhecimento, evitando o agravamento da condição de saúde, comorbidades e/ou condições que possam prejudicar a sua vida. A avaliação multidimensional inclusa nos atendimentos do enfermeiro pode ser coadjuvante do plano de atenção à saúde, dirigido para a otimização do desempenho do idoso com diabetes mellitus, favorecendo sua autonomia e independência, beneficiando sua qualidade de vida.

A escassez de registros de enfermagem é problema comum revelado em outras pesquisas, estudo desenvolvido em um hospital universitário de São Paulo buscou 
avaliar os registros de enfermagem de 424 prontuários analisados entre novembro de 2006 a janeiro de 2007, em diferentes unidades clínicas e cirúrgicas, sendo que 43,9\% destes se referiam a óbitos e $56,1 \%$ a altas. Foram verificados os seguintes itens: preenchimento do levantamento de dados; folha de centro cirúrgico; evolução de enfermagem; diagnóstico de enfermagem; prescrição de enfermagem; checagem da prescrição de enfermagem; checagem da prescrição médica, anotação de enfermagem; anotação de alta hospitalar e anotação de óbito. Os resultados revelaram que 26,7\% dos prontuários foram considerados ruins; 64,6\% regulares e 8,7\% bons (19). O estudo mostrou a escassez dos registros de enfermagem, evidenciando a necessidade de melhora dos registros.

Investigação conduzida no Hospital Público de Ensino de Natal (Rio Grande do Norte, Brasil), em julho de 2009, objetivou caracterizar os registros realizados pelo enfermeiro e demonstrar as informações presentes. Ao todo foram avaliados 30\% (23) dos prontuários que estavam em processo de faturamento, arquivo e que existiam registros. Destes, 16 (70\%) possuíam algum tipo de registro do enfermeiro, como folha de evolução, admissão em Centro Cirúrgico, altas, enquanto apenas 1 (4\%) apresentava registro do enfermeiro referente aos cuidados prestados. Estendendo a análise para a qualidade dos registros, verificou-se que 7 (30\%) estavam legíveis e 15 (65\%) apresentavam assinatura, mas não havia identificação da categoria profissional, nem a inscrição do Conselho de Enfermagem, dificultando a identificação e distinção do profissional responsável, se era enfermeiro ou técnico de enfermagem (20). Neste interim, prontuários pobres em informações podem levar a conclusões referentes ao cuidado talvez não tão positivas. A visibilidade profissional se inicia pelo registro coerente e com apanhado científico.

O código de ética dos profissionais de enfermagem aborda alguns quesitos do registro de enfermagem. Na resolução COFEN 311/2007, o Art. 25: "registrar no prontuário do paciente as informações inerentes e indispensáveis ao processo de cuidar". O Art. 68: "registrar no prontuário e em outros documentos próprios da Enfermagem informações referentes ao processo de cuidar da pessoa". Segundo o Art. 71: "incentivar e criar condições para registrar as informações inerentes e indispensáveis ao processo de cuidar". Conforme Art. 72: "registrar as informações, inerentes e indispensáveis ao processo de cuidar de forma clara, objetiva e completa" (21). O prontuário é documento legal, sendo que os registros somente terão valor se forem datados e assinados e, evidentemente, se forem legíveis e não apresentarem rasuras (22).

Os registros devem ser apreciados como proteção do profissional e do paciente. Sendo assim, ressalta-se a importância dos registros de enfermagem como forma de segurança do paciente e proteção legal do enfermeiro.

Como limitação deste estudo, destaca-se a realização da pesquisa a partir de uma única realidade, sugerindo-se que novos estudos devam ser conduzidos em outras UBS, vinculadas a outros cenários de cuidado.

\section{Conclusões}

O presente estudo permitiu identificar os itens da avaliação multidimensional do idoso utilizado nas práticas dos enfermeiros através dos registros. O estudo revelou a falta de registros de enfermagem como problema, gerando invisibilidade ao profissional e dificuldade de acompanhamento da condição de saúde e doença dos idosos. Os prontuários pobres em informações podem levar a conclusões equivocadas referentes ao cuidado, como por exemplo, à possibilidade de não prestação de atendimento ou negligência. Os registros devem ser apreciados como proteção para o profissional e 
idosos. Sendo assim, reforça-se a importância dos registros de enfermagem como forma de segurança e proteção legal.

O uso da avaliação multidimensional do idoso como ferramenta que ajuda na tomada de decisão no cuidado de enfermagem na Atenção Primária à Saúde enriquece o processo de avaliação, tratamento e cuidado do idoso. A avaliação multidimensional possibilita avaliação e implementação de medidas que podem evitar que a perda da autonomia, mantendo a independência e atividade, reforça-se o idoso saudável é financeiramente mais benéfico para o sistema de saúde. A avaliação multidimensional do idoso também possibilita a detecção precoce de vulnerabilidades ou problemas de saúde, bem como identificação de agravos, previsão de desfechos em relação a aspectos do envelhecimento. Em especial, o atendimento de enfermagem com uso da avaliação multidimensional promove estratégias de atenção à saúde com potencial para a qualidade de vida do idoso com diabetes mellitus.

Os resultados do presente estudo foram apresentados aos trabalhadores do referido local de estudo e a Secretaria Municipal de Saúde do município, através de um compilado dos principais achados, indicando as potencialidade e fragilidades identificadas, bem como recomendação de utilização da avaliação multidimensional da pessoa idosa na Atenção Básica à Saúde.

\section{Agradecimentos}

Ao curso de Enfermagem dada Universidade Federal de Santa Catarina. Ao Laboratório de Pesquisas e Tecnologias em Enfermagem, Cuidado em Saúde a Pessoas Idosas (GESPI). À Secretaria Municipal de Saúde de São José, Santa Catarina.

\section{Conflito de interesse}

Os autores declaram não haver conflito de interesse.

\section{Referências}

1. Miranda GMD, Mendes ACG, Silva ALA. O envelhecimento populacional brasileiro: desafios e consequências sociais atuais e futuras. Rev Bras Geriatr Gerontol 2016; 19(3): 507-19

2. Pimenta FB, Pinho L, Silveira MF, Botelho ACC. Fatores associados a doenças crônicas em idosos atendidos pela Estratégia de Saúde da Família. Ciênc Saúde Coletiva 2015; 20(8): 2489-98

3. Oliveira JEP, Vencio S. Diretrizes da Sociedade Brasileira de Diabetes (2015-2016). [Internet]. São Paulo: A.C. Farmacêutica; 2016 [citado em 2020 Ago 22]. Disponível em: https://www.diabetes.org.br/profissionais/images/docs/DIRETRIZES-SBD-2015-2016.pdf

4. Brasil. Ministério da Saúde. Secretaria de Atenção à Saúde. Departamento de Atenção Básica. Envelhecimento e saúde da pessoa idosa. Brasília: Ministério da Saúde; 2006

5. Clares JWB, Guedes MVC, Silva LF, Nóbrega MML, Freitas MC. Subset of nursing diagnoses for the elderly in Primary Health Care. Rev Esc Enferm USP 2016; 50(2): 272-8

6. Rossaneis MA, Haddad MCFL, Mathias TAF, Marcon SS. Differences in foot self-care and lifestyle between men and women with diabetes mellitus. Rev Latino-Am Enfermagem 2016; 24: e2761

7. Almeida ANF, Cárdenas AMC. Caracterização epidemiológica de pacientes com Diabetes Mellitus tipo 2 de uma Unidade Básica de Saúde de Macapá - AP - Brasil. Rev Ciênc Amazônia 2013; 1(1): 74

8. Santos AD, da Silva CRA, Medeiros JD, Panazzolo GLG, da Silva HCTA, Rosa Filho AAM, Teodózio ASO, Rocha MQ, dos Reis RP. Perfil epidemiológico de pacientes com diabetes mellitus. BJSCR 2018; 24(2): 40-6

9. Moreschi C, Rempel C, Siqueira DF, Backes DS, Pissaia LF, Grave MTQ. Estratégias Saúde da Família: perfil/qualidade de vida de pessoas com diabetes. Rev Bras Enferm 2018; 71(6): 2899-2906 
10. Fagundes CN, Corso ACT, González-Chica DA. Perfil epidemiológico de hipertensos e diabéticos cadastrados na Atenção Básica em Saúde, Florianópolis - SC. Rev Pesq Saúde 2017; 18(1): 28-34

11. American Diabetes Association. Standards of medical care in diabetes - 2011. Diabetes Care 2011; 34(supp. 1): 11-61

12. Cortez DN, Reis IA, Souza DAS, Macedo MML, Torres HC. Complicações e o tempo de diagnóstico do diabetes mellitus na atenção primária. Acta Paul Enferm 2015; 28(3): 250-5

13. Pereira R. A relação entre dislipidemia e diabetes mellitus tipo 2. Cadernos UniFOA 2011; 6(17): 89-94

14. Aguiar APS, Oliveira MR, Barreto NAP, Ferreira SFV, Ferreira CMM, Pinho L. Hipertensão e dislipidemia em pacientes com diabetes mellitus tipo 2: uma revisão integrativa. Montes Claros, $8^{\circ}$ Fórum de Ensino Pesquisa Extensão e Gestão; 2014.

15. Martinez LRC, Murad N. Hipertensão, diabetes e dislipidemia - mecanismos envolvidos. Rev Bras Hipertens 2014; 21(2): 92-7

16. Santos SSC, Cavalheiro BC, Silva BT, Barlem ELD, Feliciani AM, Valcarenghi RV. Avaliação multidimensional do idoso por enfermeiros brasileiros: uma revisão integrativa. Cienc Cuid Saude 2010; 9(1): 129-36

17. Sirena SA. Avaliação multidimensional do idoso: uma abordagem em atenção primária à saúde [tese de doutorado]. Porto Alegre: Faculdade de Medicina da Pontifícia Universidade Católica do Rio Grande do Sul, Curso de Medicina; 2002

18. Setz VG, D’Innocenzo M. Avaliação da qualidade dos registros de enfermagem no prontuário por meio da auditoria. Acta Paul Enferm 2009; 22(3): 313-7

19. Pedrosa KKA, Souza MFG, Monteiro AI. O enfermeiro e o registro de enfermagem em um hospital público de ensino. Rev Rene 2011; 12(3): 568-73

20. Conselho Federal de Enfermagem. Resolução Cofen 311/2007: código de ética da enfermagem. São Paulo; 2007

21. Conselho Federal de Enfermagem. Portaria n. 523/2015: guia de recomendações para registro de enfermagem no prontuário do paciente e outros documentos de enfermagem. São Paulo; 2011. 